\title{
Ahlhaus, Svenja (2020): Die Grenzen des Demos. Mitgliedschaftspolitik aus postsouveräner Perspektive
}

\author{
Frankfurt/New York: Campus. 278 Seiten. 34,95€
}

\author{
Eva-Maria Schäfferle
}

Angenommen: 31. März 2021 / Online publiziert: 26. April 2021

(C) Der/die Autor(en) 2021

1972 schrieb Robert Dahl in „After the Revolution?“: „Strange as it may seem to you, how to decide who legitimately make up , the people " - or rather $a$ people - and hence are entitled to govern themselves in their own association is a problem almost totally neglected by all the great political philosophers who write about democracy“. Knapp 50 Jahre nach Entdeckung des sogenannten „Boundary Problem“ trifft diese Aussage, zumindest auf zeitgenössische Denkerinnen, kaum noch zu. Die Frage nach der Legitimität des Volkes, nach seiner rechtmäßigen Zusammensetzung und Begrenzung, gehört heute sicherlich zu den umstrittensten und spannendsten Fragen der Demokratietheorie und wurde damit naturgemäß zum Gegenstand zahlreicher Publikationen. Warum also noch ein Buch über das Boundary Problem?

In ihrer Monografie „,Die Grenzen des Demos. Mitgliedschaftspolitik aus postsouveräner Perspektive“ gibt Svenja Ahlhaus eine so simple wie überzeugende Antwort: Weil die meisten bisherigen Beiträge der paradoxen Struktur des Problems nur unzureichend auf den Grund gehen. Schließlich stellt das Boundary Problem nicht nur die Legitimität bestehender Grenzen und damit die Legitimität bestehender Völker infrage. Es stellt die Legitimationsfrage auf einem grundlegenderen Niveau und streut damit Zweifel an der Legitimierbarkeit von Grenzen an sich. Wenn zuerst ein Volk existieren muss, bevor demokratische Entscheidungen getroffen werden können, wie kann dann demokratisch über die Zusammensetzung des Volkes entschieden werden? Und wenn kein Volk demokratische Legitimität beanspruchen kann, was berechtigt dann existierende Völker unilateral über ihre Grenzen und damit über Aufnahme und Abweisung zu entscheiden?

E.-M. Schäfferle ( $\square)$

Fachbereich Gesellschaftswissenschaften, Goethe Universität Frankfurt am Main, Frankfurt am Main, Deutschland

E-Mail: Schaefferle@soz.uni-frankfurt.de 
Im Gegensatz zum dominanten Ansatz liberaler Theoretikerinnen geht es Ahlhaus also weniger um die Suche nach dem geeignetsten Inklusionsprinzip und damit nach der besten Reform bestehender Mitgliedschaftsregime. Im Zentrum ihrer Untersuchung steht vielmehr die Frage nach dem legitimen Entscheidungsorgan und damit die Frage, wer an der Auswahl und Umsetzung entsprechender Reformen beteiligt sein muss. Das „Dogma souveräner Mitgliedschaftspolitik“ (S. 19), nach welchem bestehende Völker unilateral über ihre Grenzen entscheiden können, lehnt Ahlhaus dabei konsequent ab. Da Fragen der Mitgliedschaft und damit Entscheidungen über Inklusion und Exklusion nicht nur die Freiheit von Mitgliedern, sondern auch jene von Nichtmitgliedern beschränken, müssen sie - um demokratischen Ansprüchen zu genügen - die Interessen beider Gruppen berücksichtigen. Das Legitimitätsproblem, das Ahlhaus zu lösen versucht, ist somit ein doppeltes. Reformen der Mitgliedschaft müssen nicht nur so gestaltet sein, dass sie die Autonomierechte von Nichtmitgliedern respektieren, sondern müssen Letztere auch in den Prozess ihrer Genese einbeziehen (S. 129). Doch wie lässt sich die unvermeidbare Spannung zwischen prozeduralen und substanziellen Legitimationskriterien auflösen? Eine Antwort findet Ahlhaus in der deliberativen Demokratietheorie. Auf den Arbeiten von Jürgen Habermas, Nancy Fraser und Seyla Benhabib aufbauend, entwickelt sie einen zweigleisigen Lösungsansatz. Die prozedurale Legitimität von Mitgliedschaftsentscheidungen soll dabei durch eine geloste „Boundary Assembly“ (S. 220) garantiert werden, die paritätisch aus Mitgliedern und Nichtmitgliedern zusammengesetzt ist und verbindliche Entscheidungen über alle mitgliedschaftsrelevanten Fragen trifft. Um dem liberalen Einwand eines reinen Prozeduralismus, der Grundrechte auf dem Altar der Demokratie zu opfern bereit sei, vorzubeugen, ergänzt Ahlhaus ihre Boundary Assembly durch ein „Global Membership Law“ (S. 235), das die völkerrechtlichen Grenzen lokal getroffener Mitgliedschaftsentscheidungen absteckt.

Ahlhaus' Vorschlag kann das Boundary Problem nicht lösen - ein Anspruch, den die Autorin allerdings auch gar nicht erhebt. In der Tradition der deliberativen Demokratietheorie strebt sie nicht nach einem normativen Sollzustand, sondern nach einer schrittweisen Demokratisierung des Istzustands. Die von ihr entwickelte Boundary Assembly liefert dafür zweifelsohne wichtige Impulse. Gleichzeitig wirft sie jedoch selbst eine Reihe neuer Legitimationsfragen auf, die der kritischen Untersuchung bedürfen.

Erste Fragen resultieren aus dem nur vage umrissenen Kompetenzbereich des neuen Entscheidungsorgans. Zwar schränkt Ahlhaus diesen auf Belange der Mitgliedschaft ein, legt die Entscheidung, wann genau es sich um solche handelt, jedoch in die Hände nicht gewählter Institutionen wie Ombudspersonen oder auch der Boundary Assembly selbst (S. 234). Gerade angesichts der Komplexität von Mitgliedschaftsfragen entsteht dadurch die Gefahr einer progressiven Kompetenzverlagerung zugunsten des gelosten Gremiums, was letzten Endes sogar seiner Gründungsidee zuwiderlaufen könnte. Nichtmitglieder hätten nämlich selbst dann weitreichende Entscheidungsbefugnisse, wenn ihnen die Boundary Assembly politische Teilhaberechte im Gastland abspricht.

Aber auch die übergeordnete Ebene des Global Membership Law scheint, entgegen Ahlhaus' Annahme, nicht frei von Legitimationsanforderungen zu sein. Schließlich schließt es neben bestehendem Völkerrecht auch demokratische Inklusionsprin- 
zipien ein, was im Verbot von Ausbürgerungen deutlich wird. Nicht Staatenlosigkeit, sondern das Unterworfenheitsprinzip wird hier als primärer Grund genannt (S. 241). Wenn die Anwendung und Interpretation von Inklusionsprinzipien jedoch, wie Ahlhaus im nationalen Rahmen fordert, der demokratischen Zustimmung bedarf, bleibt unklar, warum die gleichen Legitimationsmaßstäbe nicht auch auf globaler Ebene gelten.

Abschließend lässt die partielle Behandlung des Boundary Problem, die Fragen der Einwanderung ausklammert, Zweifel an einer vollständigen Abkehr vom Dogma souveräner Mitgliedschaftspolitik aufkommen. Genau wie Benhabib scheint Ahlhaus der dem Dogma zugrunde liegenden Dichotomie zwischen „uns“ und ,ihnen“ verhaftet zu bleiben. Es geht ihr schließlich um die Korrektur eben unserer Grenzen, um die Rechte, die wir Nichtmitgliedern zugestehen. Das Boundary Problem lädt jedoch dazu ein, genau diese Dichotomie infrage zu stellen. Es fordert also in der Tat nach globalen Entscheidungsprozessen, die, wie Ahlhaus annimmt, jedoch nicht mit dem Fortbestand politischer Gemeinschaften unvereinbar sein müssen. Sobald wir anerkennen, dass wir immer beides sind, d. h. Mitglieder zu Hause und Nichtmitglieder in der Fremde, dass wir also die konfligierenden Interessen, die Ahlhaus „uns“ und ,ihnen“ zuschreibt, in unserer eigenen Person vereinen, werden globale Verfahren denkbar, die kollektive und individuelle Freiheitsrechte gleichermaßen schützen.

Das Boundary Problem bleibt ein komplexes Problem. Es zu lösen scheint unmöglich. Dass es sich trotzdem lohnt, seiner Komplexität auf den Grund zu gehen, um Transformationspotenziale zu erkennen, macht Ahlhaus in „Die Grenzen des Demos" deutlich.

Funding Open Access funding enabled and organized by Projekt DEAL.

Open Access Dieser Artikel wird unter der Creative Commons Namensnennung 4.0 International Lizenz veröffentlicht, welche die Nutzung, Vervielfältigung, Bearbeitung, Verbreitung und Wiedergabe in jeglichem Medium und Format erlaubt, sofern Sie den/die ursprünglichen Autor(en) und die Quelle ordnungsgemäß nennen, einen Link zur Creative Commons Lizenz beifügen und angeben, ob Änderungen vorgenommen wurden.

Die in diesem Artikel enthaltenen Bilder und sonstiges Drittmaterial unterliegen ebenfalls der genannten Creative Commons Lizenz, sofern sich aus der Abbildungslegende nichts anderes ergibt. Sofern das betreffende Material nicht unter der genannten Creative Commons Lizenz steht und die betreffende Handlung nicht nach gesetzlichen Vorschriften erlaubt ist, ist für die oben aufgeführten Weiterverwendungen des Materials die Einwilligung des jeweiligen Rechteinhabers einzuholen.

Weitere Details zur Lizenz entnehmen Sie bitte der Lizenzinformation auf http://creativecommons.org/ licenses/by/4.0/deed.de. 